\title{
CERN LEP indications for two light Higgs bosons and the $\mathrm{U}(1)^{\prime}$ model
}

\author{
Durmuş A. Demir, ${ }^{1}$ Levent Solmaz, ${ }^{1,2}$ and Saime Solmaz ${ }^{2}$ \\ ${ }^{1}$ Department of Physics, Izmir Institute of Technology, IZTECH, TR35430, Turkey \\ ${ }^{2}$ Department of Physics, Balıkesir University, Bal1kesir, TR10100, Turkey \\ (Received 13 September 2005; revised manuscript received 12 December 2005; published 9 January 2006)
}

\begin{abstract}
Reanalyses of LEP data have shown preference to two light $C P$-even Higgs bosons. We discuss implications of such a Higgs boson spectrum for the minimal supersymmetric model extended by a standard model singlet chiral superfield and an additional Abelian gauge invariance [the $\mathrm{U}(1)^{\prime}$ model]. We, in particular, determine parameter regions that lead to two light $C P$-even Higgs bosons while satisfying existing bounds on the mass and mixings of the extra vector boson. In these parameter regions, the pseudoscalar Higgs is found to be nearly degenerate in mass with either the lightest or next-to-lightest Higgs boson. Certain parameters of the $\mathrm{U}(1)^{\prime}$ model such as the effective $\mu$ parameter are found to be significantly bounded by the LEP two light Higgs signal.
\end{abstract}

DOI: 10.1103/PhysRevD.73.016001

PACS numbers: 11.30.Ly, 11.30.Pb, 12.60.Cn

\section{INTRODUCTION}

Supersymmetric models, in particular, the minimal supersymmetric model (MSSM) have been introduced to solve the gauge hierarchy problem of the standard model (SM). However, the MSSM itself suffers from a naturalness problem concerning the Higgsino Dirac mass nested in the superpotential of the model. This problem, the $\mu$ problem [1], has been the main source of motivation for extending the MSSM. The point is to replace $\mu$ by a chiral superfield whose scalar component develops a vacuum expectation value to induce an effective $\mu$ parameter at the desired scale. Up to now, there have been two basic models in this direction: the $\mathrm{U}(1)^{\prime}$ models (see the reviews [2]) and next-to-minimal supersymmetry i.e. NMSSM (see [3]). Both models have interesting phenomenological implications ranging from rare decays to Higgs phenomenology. In this work, we are primarily interested in the Higgs sector of the $\mathrm{U}(1)^{\prime}$ models. If one is to find an explanation for why $\mu$ parameter (having no relation to soft-breaking sector of the theory) in the MSSM is stabilized at the weak scale then such extensions of the MSSM seem to offer a phenomenologically viable pathway.

The $\mathrm{U}(1)^{\prime}$ models forbid a bare $\mu$ parameter via the additional Abelian gauge invariance, $\mathrm{U}(1)^{\prime}$ symmetry. The model predicts an additional neutral vector boson, $Z^{\prime}$, which mediates neutral currents and which mixes with the $Z$ boson of the MSSM. There are continuing collider searches for this extra $Z$ boson, each leading to certain bounds on its couplings and mass [4]. There is a host of constraints originating from different observables [5]. The most important and direct ones concern bounds on $Z^{\prime}$ mass and strength of mixing between $Z$ and $Z^{\prime}$ bosons.

The $\mathrm{U}(1)^{\prime}$ models generically predict an additional $C P$-even Higgs boson which typically weighs near $Z^{\prime}$. The rest are similar to those in the MSSM in terms of their overall scale and dependencies on the electroweak Higgs doublets (see $[6,7]$ for instance).
The recent reanalysis [8] of the LEP data by all four LEP collaborations has given an indication for two, rather than one, light Higgs bosons. Although it is not a clear enough signal to state the existence of two light Higgs bosons in the bulk of LEP data, all four LEP experiments see a mild excess near $98 \mathrm{GeV}$ with significance of 2.3 standard deviations to be contrasted with the second signal seen at $114 \mathrm{GeV}$ at 1.7 standard deviations. This two light Higgs signal has been interpreted within the framework of the MSSM in [9-11]. In the MSSM, if the lightest and next-tolightest Higgs bosons are to explain the data the overall scale of the Higgs sector turns out to be rather close to $M_{Z}$ (as will be seen, this does not have to be so in $\mathrm{U}(1)^{\prime}$ models).

The purpose of this work is to determine the implications of the LEP two light Higgs signal within $\mathrm{U}(1)^{\prime}$ models in which the $\mu$ parameter of the MSSM is dynamically generated. The paper is organized as follows. In Sec. II we give a brief overview of the $\mathrm{U}(1)^{\prime}$ model. In Sec. III we discuss bounds on mass and mixings of the $Z^{\prime}$ boson. In Sec. IV we discus the LEP two light Higgs signal along with its MSSM and U(1)' interpretations. In Sec. V we provide a thorough analysis of several observables, especially the couplings and masses of the Higgs bosons, by a scan of the parameter space. We conclude in Sec. VI.

\section{OVERVIEW OF THE U(1 $)^{\prime}$ MODELS}

In addition to the ones in the SM, there can exist new gauge bosons weighing around a $\mathrm{TeV}$ provided that they are sufficiently heavy or weakly coupled to the observed matter. Neutral, color-singlet gauge bosons, the $Z^{\prime}$ bosons, can arise as low-energy manifestations of grand unified theories (GUT) [12], strings [13], or dynamical electroweak breaking [14] theories. In this work we will study a minimal $\mathrm{U}(1)^{\prime}$ model (in that it differs from the MSSM only by an additional U(1) invariance and by the presence of a single MSSM singlet chiral superfield $S$, to be contrasted with models involving a number of singlets or 
exotics [7,15]) described in [6] without referring to its origin. The model is based on the gauge group

$$
\mathrm{SU}(3)_{c} \times \mathrm{SU}(2)_{L} \times \mathrm{U}(1)_{Y} \times \mathrm{U}(1)^{\prime},
$$

with gauge couplings $g_{3}, g_{2}, g_{Y}, g_{Y^{\prime}}$, respectively. The matter content includes the MSSM superfields and a SM singlet $S$, which are all generically assumed to be charged under the additional $U(1)^{\prime}$ gauge symmetry. Explicitly, the particle content is $\hat{L}_{i} \sim\left(1,2,-1 / 2, Q_{L}\right), \quad \hat{E}_{i}^{c} \sim$ $\left(1,1,1, Q_{E}\right), \hat{Q}_{i} \sim\left(3,2,1 / 6, Q_{Q}\right), \hat{U}_{i}^{c} \sim\left(\overline{3}, 1,-2 / 3, Q_{U}\right)$, $\hat{D}_{i}^{c} \sim\left(\overline{3}, 1,1 / 3, Q_{D}\right), \quad \hat{H}_{d} \sim\left(1,2,-1 / 2, Q_{H_{d}}\right), \quad \hat{H}_{u} \sim$ $\left(1,2,1 / 2, Q_{H_{u}}\right), \hat{S} \sim\left(1,1,0, Q_{S}\right)$, in which $i$ is the family index.

The superpotential includes a Yukawa coupling of the two electroweak Higgs doublets $H_{u, d}$ to the singlet $S$ as well as the top quark Yukawa coupling:

$$
W=h_{s} \hat{S} \hat{H}_{u} \cdot \hat{H}_{d}+h_{t} \hat{U}_{3}^{c} \hat{Q}_{3} \cdot \hat{H}_{u},
$$

whose gauge invariance under $U(1)^{\prime}$ requires that $Q_{H_{u}}+$ $Q_{H_{d}}+Q_{S}=0$ and $Q_{Q_{3}}+Q_{U_{3}}+Q_{H_{u}}=0$. Appearance of a bare $\mu$ parameter in the superpotential is completely forbidden as long as $Q_{S} \neq 0$. In analyzing the model we will always impose this constraint on charges.

In (2) we have kept only the top quark Yukawa coupling. The neglect of all the light fermion contributions to the superpotential, especially those of the bottom quark and tau lepton, is justified as long as we remain in low $\left\langle H_{u}\right\rangle /\left\langle H_{d}\right\rangle \equiv \tan \beta$ domain so that hierarchy of the fermion masses (e.g. $m_{b} / m_{t}$ ) is generated by the corresponding Yukawa couplings themselves.

As we are primarily interested in the third family, in what follows we shall suppress the family index i.e. we take $Q_{Q_{3}} \equiv Q_{Q}, Q_{U_{3}} \equiv Q_{U}$, and $Q_{D_{3}} \equiv Q_{D}$. The softbreaking terms relevant for our analysis are given by

$$
\begin{aligned}
-\mathcal{L}_{\text {soft }} \ni & \left(A_{s} h_{s} S H_{u} \cdot H_{d}+A_{t} h_{t} \tilde{U}^{c} \tilde{Q} \cdot H_{u}+\text { H.c. }\right) \\
& +m_{u}^{2}\left|H_{u}\right|^{2}+m_{d}^{2}\left|H_{d}\right|^{2}+m_{s}^{2}|S|^{2}+M_{\tilde{Q}}^{2}|\tilde{Q}|^{2} \\
& +M_{\tilde{U}}^{2}|\tilde{U}|^{2}+M_{\tilde{D}}^{2}|\tilde{D}|^{2},
\end{aligned}
$$

where $A_{s}$ and $A_{t}$ are holomorphic trilinear couplings pertaining to Higgs and stop sectors, respectively. Clearly, there is no reason to expect them to be universal at the weak scale even if they are at the MSSM GUT scale [6]. In general, the gaugino masses and soft trilinear couplings $A_{s, t}$ of (3) can be complex; if so, they can provide sources of $C P$ violation (without loss of generality, the Yukawa couplings $h_{s, t}$ can be assumed to be real). However, for simplicity and definiteness we take all soft parameters real i.e. we restrict our discussions to $C P$-conserving theory.

The model at hand provides a dynamical origin for certain parameters in the MSSM Higgs sector. Indeed, below the scale of $\mathrm{U}(1)^{\prime}$ breakdown the $\mu$ parameter of the MSSM is induced to be

$$
\mu_{\mathrm{eff}}=h_{s}\langle S\rangle \equiv \frac{h_{s}}{\sqrt{2}} v_{s}
$$

where the Higgs bilinear soft mass $B$ of the MSSM is given by

$$
B_{\text {eff }}=\mu_{\text {eff }} A_{s} .
$$

These effective parameters suggest that MSSM is an effective theory to be completed by $\mathrm{U}(1)^{\prime}$ gauge invariance with a chiral superfield $S$ above $\langle S\rangle \equiv v_{s} / \sqrt{2}$.

Going back to the superpotential (2), the truncation of the Yukawa sector to top quark Yukawa interaction rests on the assumption that $\tan \beta$ does not rise to large values. Notably, in $\mathrm{U}(1)^{\prime}$ models $\tan \beta \sim 1$ is not disfavored if not preferred. (As an example, one recalls that the "large trilinear coupling minimum" - the minimum of the potential that occurs when trilinear couplings are hierarchically larger than the soft mass-squareds of the Higgs fieldswhich has been extensively studied in $[6,15]$ exhibits a strong preference to $\tan \beta \simeq 1$. However, this is no more than an example of existence. In fact, according to existing bounds $Z^{\prime}$ boson is to weigh well above the $Z$ bosonunless certain specific assumptions e.g. leptophobicity are not made - and thus this specific minimum is not expected to arise in our analysis.) In what follows we will take $\tan \beta$ to be close to unity when scanning the parameter space.

The $Z$ and $Z^{\prime}$ bosons acquire their masses by eating, respectively, $\quad \operatorname{Im}\left[-\sin \beta H_{u}^{0}+\cos \beta H_{d}^{0}\right] \quad$ and $\operatorname{Im}\left[\cos \alpha \cos \beta H_{u}^{0}+\cos \alpha \sin \beta H_{d}^{0}-\sin \alpha S\right]$ where

$$
\cot \alpha=\frac{v}{v_{s}} \sin \beta \cos \beta,
$$

and $v^{2}=v_{u}^{2}+v_{d}^{2}$ with $v_{u}^{2} / 2 \equiv\left\langle H_{u}^{0}\right\rangle^{2}, \quad v_{d}^{2} / 2 \equiv\left\langle H_{d}^{0}\right\rangle^{2}$. Clearly, as $v_{s} \rightarrow \infty, \alpha \rightarrow \pi / 2$. The remaining neutral degrees of freedom $\mathcal{B}=\left\{\operatorname{Re}\left[H_{u}^{0}\right]-\left\langle H_{u}^{0}\right\rangle, \operatorname{Re}\left[H_{d}^{0}\right]-\right.$ $\left\langle H_{d}^{0}\right\rangle, \operatorname{Re}[S]-\langle S\rangle, \operatorname{Im}\left[\sin \alpha \cos \beta H_{u}^{0}+\sin \alpha \sin \beta H_{d}^{0}+\right.$

$\cos \alpha S]\}$ span the space of massive scalars. The physical Higgs bosons are defined by

$$
H_{i}=\mathcal{R}_{i j} \mathcal{B}_{j}
$$

where the mixing matrix $\mathcal{R}$ necessarily satisfies $\mathcal{R} \mathcal{R}^{T}=$ 1 , and it has already been computed up to one-loop order in [6,16-18]. In the $C P$-conserving limit the theory contains three $C P$-even, one $C P$-odd, and a charged Higgs boson. We will name physical $C P$-even states as $H_{1}=h, H_{2}=$ $H$, and $H_{3}=H^{\prime}$ with $m_{h}<m_{H}<m_{H}^{\prime}$, and the $C P$-odd one as $H_{4}=A$ with mass $m_{A}$. Clearly, $m_{A}^{2}$ grows with growing $A_{s} v_{s}$ yet this tree-level expectation is modified by radiative corrections. At tree level, the lightest Higgs boson mass is bounded as

$$
\begin{aligned}
m_{h}^{2} \leq & M_{Z}^{2} \cos ^{2} 2 \beta+\frac{1}{2} h_{s}^{2} v^{2} \sin ^{2} 2 \beta+g_{Y}^{\prime 2}\left(Q_{H_{d}} \cos ^{2} \beta\right. \\
& \left.+Q_{H_{u}} \sin ^{2} \beta\right)^{2} v^{2},
\end{aligned}
$$

where the first term on the right-hand side is nothing but the MSSM bound where the lightest Higgs is lighter than 
the $Z$ boson at tree level. The second term is a $F$ term contribution that also exists in the NMSSM. The last term, the $\mathrm{U}(1)^{\prime} D$ term contribution, enhances the upper bound in proportion with $g_{Y}^{\prime 2}$. Hence, rather generically, the $\mathrm{U}(1)^{\prime}$ models are the ones admitting largest $m_{h}$ at tree level. This property is highly advantageous for accommodating relatively large values of $m_{h}$ as there is no need for large radiative corrections. Indeed, for $m_{h} \sim 114 \mathrm{GeV}$, for instance, one needs sizeable radiative corrections in the MSSM whereas in $\mathrm{U}(1)^{\prime}$ models this is not needed at all $[17,19]$. However, when $m_{h}$ tends to take smaller values, tree- and loop-level contributions to $m_{h}$ must conspire to generate $m_{h}$ correctly. Hence, in a small $m_{h}$ regime the most severely constrained model [among MSSM, NMSSM and $\mathrm{U}(1)^{\prime}$ models] turns out to be the $\mathrm{U}(1)^{\prime}$ model. In this sense, one expects the LEP two light Higgs signal to bound certain parameters of the $\mathrm{U}(1)^{\prime}$ models in a significant way. For example, when $m_{h}$ varies in a certain interval $h_{s}$ is expected to remain within a certain bound depending on the size of the $\mathrm{U}(1)^{\prime} D$ term contribution, as suggested by (8). The discussions in Sec. V will provide a detailed analysis of the constraints on $\mathrm{U}(1)^{\prime}$ models from LEP II data by taking into account the radiative corrections to the Higgs sector. In what follows we will base all estimates on one-loop Higgs boson masses and mixings computed in [16]. In the next section we will discuss certain phenomenological bounds on mass and couplings of the $Z^{\prime}$ boson to determine the available parameter space.

\section{CONSTRAINTS FROM $Z-Z^{\prime}$ MIXING}

The $Z^{\prime}$ boson couples to neutral currents of MSSM fields with a strength varying with the $\mathrm{U}(1)^{\prime}$ gauge coupling and $\mathrm{U}(1)^{\prime}$ charge of fields. Currently, the main constraints on the existence of a $Z^{\prime}$ boson stem from: (i) precision data on neutral current processes, (ii) modifications in $Z$ boson couplings due to its mixing with $Z^{\prime}$ on and off the $Z$ pole, and (iii) direct searches at high energy colliders. Current bounds carry an unavoidable model dependence since a TeV scale $Z^{\prime}$ can be of various origin [12-14]. When certain model parameters [say, $\mathrm{U}(1)^{\prime}$ gauge coupling and $\mathrm{U}(1)^{\prime}$ charges of the fields] are fixed one can derive bounds on the remaining parameters (say, $Z^{\prime}$ mass). In this section we will discuss implications of bounds on mixing between $Z$ and $Z^{\prime}$ bosons on $\mathrm{U}(1)^{\prime}$ charge assignment and electroweak breaking parameters.

Within $\mathrm{U}(1)^{\prime}$ models, the strongest constraints arise from the nonobservation to date of a $Z^{\prime}$, both from direct searches $[4,20]$ and from indirect precision tests from $Z$ pole, LEP II, and neutral weak current data [21,22]. The $Z-Z^{\prime}$ mixing is described by the mass-squared matrix ${ }^{1}$

\footnotetext{
${ }^{1}$ Our description of $Z-Z^{\prime}$ mixing is at tree level i.e. we do not include loop corrections to $Z$ and $Z^{\prime}$ masses as well as to their mixing mass. Moreover, we neglect possible kinetic mixing between $Z$ and $Z^{\prime}$ [23].
}

$$
M_{Z-Z^{\prime}}=\left(\begin{array}{cc}
M_{Z}^{2} & \Delta^{2} \\
\Delta^{2} & M_{Z^{\prime}}^{2}
\end{array}\right)
$$

where

$$
\begin{aligned}
& M_{Z}^{2}=G^{2} v^{2} / 4, \\
& M_{Z^{\prime}}^{2}=g_{Y^{\prime}}^{2}\left(Q_{H_{u}}^{2} v_{u}^{2}+Q_{H_{d}}^{2} v_{d}^{2}+Q_{s}^{2} v_{s}^{2}\right), \\
& \Delta^{2}=\frac{1}{2} g_{Y^{\prime}} G\left(Q_{H_{u}} v_{u}^{2}-Q_{H_{d}} v_{d}^{2}\right),
\end{aligned}
$$

with $G^{2}=g_{Y}^{2}+g_{2}^{2}=g_{2}^{2} / \cos ^{2} \theta_{W}$. Current bounds imply that the $Z-Z^{\prime}$ mixing angle, defined by

$$
\alpha_{Z-Z^{\prime}}=\frac{1}{2} \arctan \left(\frac{2 \Delta^{2}}{M_{Z^{\prime}}^{2}-M_{Z}^{2}}\right)
$$

should not exceed few $\times 10^{-3}$ in absolute magnitude.

Implications of a small $\alpha_{Z-Z^{\prime}}$ have already been analyzed previously $[6,21]$. One can see from (11) that unless $M_{Z^{\prime}} \gg M_{Z}$, the $Z-Z^{\prime}$ mixing angle is naturally of $\mathcal{O}(1)$. Therefore, a small $\left|\alpha_{Z-Z^{\prime}}\right|$ requires a cancellation in the mixing term $\Delta^{2}$ for a given value of $\tan \beta$. For models in which $M_{Z^{\prime}} \sim O\left(M_{Z}\right)$, this cancellation must be nearly exact. However, this tuning is alleviated when $Z^{\prime}$ mass is near its natural upper limit of a few TeV. Hence, $\tan ^{2} \beta$ must be tuned around $Q_{H_{d}} / Q_{H_{u}}$ with a precision determined by the size of $\alpha_{Z-Z^{\prime}}$ and how heavy $Z^{\prime}$ is.

In general, larger the mass of $Z^{\prime}$ the smaller the finetuning needed to suppress $\Delta^{2}$ and the less severe the impact of phenomenological bounds. For instance, the assumption of leptophobicity does not stand as a phenomenological necessity for heavy $Z^{\prime}$. Nevertheless, one should keep in mind that the heavier the $Z^{\prime}$ the more difficult it is to stabilize $\mu_{\text {eff }}$ if they are governed by the same Higgs sector. A rather interesting model which overcomes this difficulty was constructed in [15]. This model is, however, beyond the scope of this work.

For definiteness, our numerical analyses will be based on two specific U(1)' models - the model I and model II. They are differentiated by the $\mathrm{U}(1)^{\prime}$ charge assignments of the fields. For the purpose of this work, it suffices to fix charges of $H_{u}, H_{d}, S, Q$, and $U$, and they are depicted in Table I. The model I is taken from [6] where $Q_{H_{u}}$ and $Q_{H_{d}}$ were chosen to make $\tan \beta \sim 1$ appropriate for the "large trilinear coupling vacuum" mentioned in the text. Model II is taken from a recent discussion [19] of $\mathrm{U}(1)^{\prime}$ models where a family-nonuniversal charge assignment was used to cancel anomalies of the model such that those fermions whose Yukawa interactions are forbidden by the family

TABLE I. The $\mathrm{U}(1)^{\prime}$ charge assignments of the fields in model I and model II.

\begin{tabular}{lccccc}
\hline \hline & $Q_{H_{u}}$ & $Q_{H_{d}}$ & $Q_{S}$ & $Q_{Q}$ & $Q_{U}$ \\
\hline Model I & -1 & -1 & 2 & $1 / 2$ & $1 / 2$ \\
Model II & -1 & -2 & 3 & 0 & 1 \\
\hline \hline
\end{tabular}



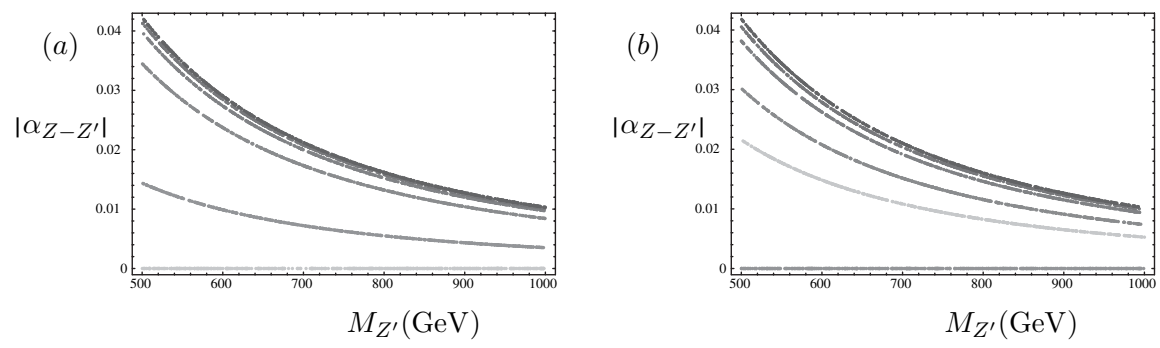

FIG. 1. Variation of the $Z-Z^{\prime}$ mixing angle with $M_{Z^{\prime}}$ for different values of $\tan \beta$. We let $M_{Z^{\prime}}$ vary from 0.5 to 1 TeV and fix $\mathrm{U}(1)^{\prime}$ charges of the Higgs fields as in model I [panel (a)] and model II [panel (b)] described in Table I. The shading of the curves is such that darkness of the curves increases as $\tan \beta$ takes values $\tan \beta=1, \sqrt{2}, 3,5,7$, and 10. The brightest curve in panel (a) and the next-tobrightest curve in panel (b) corresponds, respectively, to $\tan \beta=1$ and $\sqrt{2}$ for which $\Delta^{2}$ vanishes exactly.

dependence of the charges get their masses from nonholomorphic soft terms, radiatively. Our discussions here are restricted to holomorphic soft terms with no analysis of anomalies; hence, use of model II is, effectively, no more than a specific choice of charges.

In Fig. 1 we depict the variation of $\left|\alpha_{Z-Z^{\prime}}\right|$ with $M_{Z^{\prime}}$ for model I (left panel) and model II (right panel). The curves are for $\tan \beta=1, \sqrt{2}, 3,5,7$, and 10 whose shadings are brightest for $\tan \beta=1$ and darkest for $\tan \beta=10$. One notices that at $\tan \beta=\sqrt{Q_{H_{d}} / \bar{Q}_{H_{u}}}$ (which equals to 1 for model I and $\sqrt{2}$ for model II) the $Z-Z^{\prime}$ mixing angles vanish exactly irrespective of how heavy $Z^{\prime}$ is. However, as $\tan \beta$ departs from this specific value the mixing angle grows rapidly, and it becomes necessary to increase $M_{Z^{\prime}}$ to higher values to agree with the bound. Indeed, even for $\tan \beta=\sqrt{2}$ in model I (similarly for $\tan \beta=1$ in model II) the $Z^{\prime}$ boson has to weigh $\sim 1.5 \mathrm{TeV}$ for $\left|\alpha_{Z-Z^{\prime}}\right|$ to fall below $10^{-3}$. Therefore, restriction of $M_{Z^{\prime}}$ below a TeV necessarily enforces $\tan \beta$ to remain in close vicinity of $\sqrt{Q_{H_{d}} / Q_{H_{u}}}$. This justifies the truncation of the Yukawa sector to top quark couplings in (2).

This section completes the specification of the $\mathrm{U}(1)^{\prime}$ models to be used in the following sections and describes the impact of $Z-Z^{\prime}$ mixing angle on model parameters, in particular, on $\tan \beta$. By examining the response of certain observables to variations in charges (and various soft masses discussed in the last section) one can trace model-dependence in predictions of the theory. In the next section we will briefly discuss the LEP two light Higgs signal and its interpretation within the MSSM.

\section{LEP INDICATIONS FOR TWO LIGHT HIGGS BOSONS}

Using $e^{+} e^{-}$collision data at center-of-mass energies between 189 and $209 \mathrm{GeV}$, the search performed by all four LEP groups, ALEPH, DELPHI, L3, and OPAL collaborations, set the lower limit of $114.4 \mathrm{GeV}$ at $95 \%$ confidence level for the SM Higgs boson [8]. Interestingly, in all four experiments there is an additional common signal of a mild excess near $98 \mathrm{GeV}$. The signal around $98 \mathrm{GeV}$ is a $2.3 \sigma$ effect which should be compared with the $1.7 \sigma$ excess around $114 \mathrm{GeV}$. Notably, the former is a weaker signal than the latter, and if it is not related to background fluctuations or some other experimental uncertainties then extensions of the SM offering more than one Higgs doublet are favored. Here supersymmetric models stand as highly viable candidates. In fact, such experimental results can fit quite well to MSSM or its minimal extensions i.e. NMSSM or $\mathrm{U}(1)^{\prime}$ models. Indeed, all three of these models have $h$ (the lightest of all Higgs bosons), $H$ (the next-to-lightest Higgs), and $A$ (the $C P$-odd Higgs boson) in common. The heavier Higgs bosons are model-dependent in number and mass range. These Higgs states, if sufficiently light, can contribute significantly to the formation of four-fermion final states in $e^{-} e^{+}$collisions. In fact, supersymmetric signals $e^{+} e^{-} \rightarrow(h, H) Z$ can give significant contributions especially to two heavy fermion signals characterized by final states containing $\bar{b} b \bar{f} f$ or $\tau^{+} \tau^{-} \bar{f} f-f$ standing for a light fermion. On the other hand, associated production of opposite- $C P$ Higgs bosons, $e^{+} e^{-} \rightarrow(h, H) A$, can contribute to four heavy fermion events characterized by final states consisting of $\bar{b} b \bar{f} f$ or $\tau^{+} \tau^{-} \bar{f} f-f$ standing for $b$ quark or $\tau$ lepton. Of course, both signals suffer form backgrounds generated by $Z$ boson decays into $\bar{b} b$ and $\tau^{+} \tau^{-}$.

The MSSM interpretation of the LEP signal [8] has been considered already in $[9,10,24]$. The main implication of this two light Higgs signal is that the MSSM Higgs sector must be light as a whole i.e. it should not enter the decoupling regime where $m_{H} \sim m_{A} \gg m_{h}$. In fact, as has been emphasized in [10], the main idea is to identify the signal at $98 \mathrm{GeV}$ with $h$ and the one at $114 \mathrm{GeV}$ with $H$. This identification is justified as long as $h Z Z$ coupling is sufficiently suppressed to cause a relatively weak signal at $98 \mathrm{Gev}$. This indeed happens if the overall mass scale of the Higgs sector is close to $M_{Z}$. In [9] discussions were given of various MSSM parameter regions, including finite $C P$-odd phases, predicting light Higgs bosons in the LEP data. This analysis suggests that the requisite range of the $\mu$ parameter is typically $\mathcal{O}(2 \mathrm{TeV})$ unless $m_{A} \simeq m_{h}$ within 
a few $\mathrm{GeV}$. In general, the relative phase between $A_{t}$ and $\mu$ provides an additional freedom for achieving the correct configuration. It is interesting that, according to [9], the least fine-tuned parameter space corresponds to a light Higgs boson of mass $m_{h} \simeq 114 \mathrm{GeV}$ with all the rest being heavy. (Here fine-tuning refers to sensitivity of a given parameter set to changes in parameter values specified at the GUT scale.)

The recent work [11] provides a detailed analysis of the two light Higgs signal within the $C P$-conserving MSSM by imposing bounds from $B_{d} \rightarrow X_{s} \gamma$, muon $g-2, B_{s} \rightarrow \mu \bar{\mu}$ as well as from relic density of the lightest neutralino. The allowed parameter space turns out to be particularly wide for $\mu \gtrsim 1 \mathrm{TeV}$. The bounds from these observables are found to constrain the MSSM parameter space unless model parameters are tuned to evade them [11]. Consequently, in both $C P$-conserving $[10,11]$ and $C P$-violating [9] cases the MSSM offers a wide parameter region which provides an explanation for the LEP two light Higgs signal.

In this work we will discuss possible implications of the LEP two light Higgs signal for the U(1)' models specified by charge assignments in Table I. Our analyses are based on the radiatively-corrected Higgs boson masses and mixings computed in [16]. For the model under concern to explain the data, the signal strengths must be reproduced correctly at the indicated Higgs mass values. The contribution of $Z^{\prime}$ mediation is negligible within its mass range, and thus, we focus on the $Z$ boson mediated Higgs production processes. The Higgs production cross sections depend on all the parameters in the Higgs mass-squared matrix via the Higgs boson couplings to $Z$ as well as the Higgs boson masses. Leaving their tensor structures aside, the Higgs $-Z-Z$ couplings are given by [25]

$$
\begin{aligned}
& C_{h Z Z}=\mathcal{R}^{h d} \cos \beta+\mathcal{R}^{h u} \sin \beta, \\
& C_{H Z Z}=\mathcal{R}^{H d} \cos \beta+\mathcal{R}^{H u} \sin \beta, \\
& C_{H^{\prime} Z Z}=\mathcal{R}^{H^{\prime} d} \cos \beta+\mathcal{R}^{H^{\prime} u} \sin \beta,
\end{aligned}
$$

in units of the SM $h Z Z$ coupling $G M_{Z}$. On the other hand, coupling of the opposite- $C P$ Higgs bosons to $Z$ are given by

$$
\begin{aligned}
& C_{h A Z}=\sin \alpha\left(\mathcal{R}^{h u} \cos \beta-\mathcal{R}^{h d} \sin \beta\right), \\
& C_{H A Z}=\sin \alpha\left(\mathcal{R}^{H u} \cos \beta-\mathcal{R}^{H d} \sin \beta\right), \\
& C_{H^{\prime} A Z}=\sin \alpha\left(\mathcal{R}^{H^{\prime} u} \cos \beta-\mathcal{R}^{H^{\prime} d} \sin \beta\right),
\end{aligned}
$$

in units of $G / 2$, where $G=\sqrt{g_{Y}^{2}+g_{2}^{2}}$ as defined before. Here $\mathcal{R}$ is the Higgs mixing matrix defined in Sec. II. The notation is such that $\mathcal{R}^{h d}$, for instance, denotes the entry of $\mathcal{R}$ formed by the row corresponding to lightest Higgs boson $h$ and by the column corresponding to the neutral $C P$-even component, $\phi_{d}$, of $H_{d}$. The Higgs mass matrix is taken in the basis $\mathcal{B}$ given in Sec. II.
These couplings govern what Higgs bosons are produced with what strength if they are kinematically accessible. The number of excess events around $98 \mathrm{GeV}$ forms about $10 \%$ of the events which would be generated by the SM Higgs boson production with $m_{h_{\mathrm{SM}}}=98 \mathrm{GeV}$. More quantitatively, the cross sections satisfy

$$
\frac{\sigma\left(e^{+} e^{-} \rightarrow h Z\right)}{\sigma\left(e^{+} e^{-} \rightarrow h_{\mathrm{SM}} Z\right)}=C_{h Z Z}^{2} \simeq 0.1
$$

if $m_{h}=m_{h_{\mathrm{SM}}}=98 \mathrm{GeV}$. Hence, given the statistical significances of the two signals at 98 and $114 \mathrm{GeV}$, the parameter ranges favored by the LEP excess events turn out to be

$$
\begin{aligned}
95 \mathrm{GeV} & \leq m_{h} \leq 101 \mathrm{GeV}, \\
111 \mathrm{GeV} & \leq m_{H} \leq 119 \mathrm{GeV}, \\
0.056 & \leq C_{h Z Z}^{2} \leq 0.144,
\end{aligned}
$$

as has first been derived by [10] while analyzing the signal within the MSSM. The strength of the $114 \mathrm{GeV}$ signal, with respect to the SM expectation, depends on the coupling strength of $H^{\prime}$ to the $\mathrm{Z}$ boson: $C_{H Z Z}^{2} \simeq 0.9-C_{H^{\prime} Z Z}^{2}$. However, when $Z^{\prime}$ is heavy so is $H^{\prime}$ and $C_{H Z Z}^{2}$ turns out to be rather close to the MSSM expectation. In the opposite limit i.e. when $Z^{\prime}$ weighs relatively light so is $H^{\prime}$, and $C_{H^{\prime} Z Z}^{2}$ becomes too large to allow $C_{H Z Z}^{2}$ to remain close to its MSSM counterpart. These parameter domains will be illustrated by scanning the parameter space in the next section.

Clearly, $\left(h, H, H^{\prime}\right)-Z-Z$ and $\left(h, H, H^{\prime}\right)-A-Z$ couplings are correlated with each other. The strength of correlation depends on how light $H^{\prime}$ is, that is, how close $\mathrm{U}(1)^{\prime}$ breaking scale is to $M_{Z}$. For instance, for heavy $H^{\prime}$ the singlet components of the remaining Higgs bosons are suppressed, $\alpha \rightarrow \pi / 2$, and one finds $C_{H Z Z}^{2} \simeq C_{h A Z}^{2} \simeq 0.9$. This enhances the $h A$ production compared to $H A$ production, if they are kinematically accessible. Nevertheless, one keeps in mind that productions of opposite- $C P$ Higgs bosons are $P$ wave suppressed; moreover, LEP data have not yet been subjected to a global analysis like [8] for such final states.

In the next section we will provide a scan of the $\mathrm{U}(1)^{\prime}$ parameter space to determine allowed regions and correlations among the model parameters under the LEP constraints (15).

\section{CONFRONTING U(1) ${ }^{\prime}$ MODEL WITH LEP DATA}

In this section we will determine constraints on the parameters of $\mathrm{U}(1)^{\prime}$ model from the LEP two light Higgs signal. Before imposing the LEP bounds (15), we list down allowed ranges or values of the model parameters. These choices, which stem from different reasons, bring considerable ease in scanning of the parameter space: 

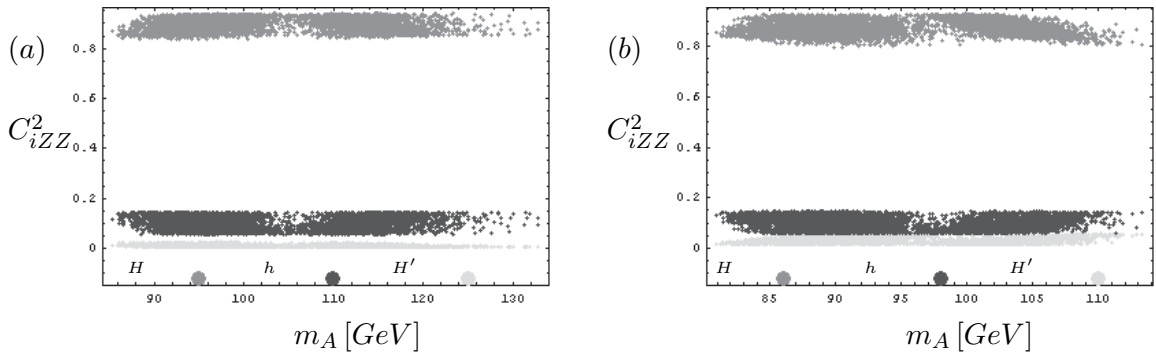

FIG. 2. Variation of $C_{\left(h, H, H^{\prime}\right) Z Z}^{2}$ with $m_{A}$ for model I [panel (a)] and model II [panel (b)] after imposing the LEP bounds (15). Obviously, $C_{H^{\prime} Z Z}^{2}$ is rather small (though it can take slightly larger values in model II than in model I) and therefore $C_{H Z Z}^{2} \approx$ $1-C_{h Z Z}^{2} \approx 0.9$. The $\mathrm{U}(1)^{\prime}$ charge assignments influence shape of the allowed domains of $C_{\left(h, H, H^{\prime}\right) Z Z}^{2}$ as well as their allowed ranges. These figures are useful also for determining the allowed range of $m_{A}: 133 \geq m_{A} \geq 86 \mathrm{GeV}$ in model I and $113 \geq m_{A} \geq 81 \mathrm{GeV}$ in model II.

(i) $M_{Z^{\prime}} \in[0.5,1] \mathrm{TeV}$. This range for $M_{Z^{\prime}}$ is chosen to agree with bounds from direct collider searches [4] on one hand and to prevent $M_{Z^{\prime}}$ slipping into deep $\mathrm{TeV}$ domain on the other hand. The latter introduces a hierarchy problem within the gauge boson sector [15,26].

(ii) $\left|\alpha_{Z-Z^{\prime}}\right| \leq 2 \times 10^{-3}$. Using this bound together with the aforementioned interval for $M_{Z^{\prime}}, \tan \beta$ is found to remain in close vicinity of $\sqrt{Q_{H_{d}} / Q_{H_{u}}}$ : $0.94 \leq \tan \beta \leq 1.06$ for model $\mathrm{I}$ and $1.36 \leq$ $\tan \beta \leq 1.47$ for model II.

(iii) $g_{Y^{\prime}}^{2}=\frac{5}{3} G^{2} \sin ^{2} \theta_{W}$. This choice for $g_{Y^{\prime}}$ might be inspired from one-step GUT breaking; however, care should be taken to the normalization of the $\mathrm{U}(1)^{\prime}$ charges. Indeed, overall normalization of the charges (as in GUTs, for instance) results in a rescaling of $g_{Y^{\prime}}$ so that the value quoted here does not need to be the correct choice for $\mathrm{U}(1)^{\prime}$ charges in Table I. Therefore, this equality for $g_{Y^{\prime}}$ should be regarded as a specific choice, not necessarily stemming from the GUTs.

(iv) $h_{s} \in[0.1,0.7]$. The RGE studies in $[6,15]$ suggest that $h_{s} \lesssim \mathcal{O}(0.7)$ for perturbativity up to the MSSM gauge coupling unification scale.

(v) $\mathrm{U}(1)^{\prime}$ charges of the fields as in Table I.

(vi) $M_{\tilde{Q}}, M_{\tilde{U}} \in[0.5,5] v, 0<A_{t, s} \lesssim 10 v$, and $m_{\tilde{t}_{1}} \geq$ $100 \mathrm{GeV}, \tilde{t}_{1}$ being the lighter stop. These choices appropriately put soft-breaking parameters within $\mathrm{TeV}$ range.

In what follows we will impose the LEP bounds (15) on this parameter space to determine allowed ranges for model parameters. This determination, depending on how tight it is, will facilitate construction of a low-energy softly-broken supersymmetric theory devoid of the $\mu$ problem.

We start the analysis by plotting various Higgs- $Z$ coupling-squareds with respect to the pseudoscalar Higgs mass $m_{A}$ by applying the LEP bounds in (15). $C_{\left(h, H, H^{\prime}\right) Z Z}^{2}$ are shown in Fig. 2 and $C_{\left(h, H, H^{\prime}\right) A Z}^{2}$ in Fig. 3 (the shading of each figure is described by the inset in the panels). This analysis proves useful for determining the (experimentally unconstrained) range of $m_{A}$. Indeed, as suggested by the figures, $133 \geq m_{A} \geq 86 \mathrm{GeV}$ in model I and $113 \geq m_{A} \geq$ $81 \mathrm{GeV}$ in model II. These figures enable one to determine the correlations among various Higgs- $Z$ couplings. First of all, $C_{H^{\prime} Z Z}^{2} \ll 1$ and $C_{H^{\prime} Z Z}^{2} \lesssim C_{h Z Z}^{2}$ for all parameter values of interest. Therefore, $C_{H Z Z}^{2} \simeq 0.9-C_{H^{\prime} Z Z}^{2} \approx 0.9$ as was discussed in Sec. IV. Furthermore, as comparison of Figs. 2 and 3 reveals, $C_{h A Z}^{2} \simeq C_{H Z Z}^{2}, C_{H A Z}^{2} \simeq C_{h Z Z}^{2}$, and $C_{H^{\prime} A Z}^{2} \simeq$ $C_{H^{\prime} Z Z}^{2}$. Clearly, these correlations among the couplings become precise when $M_{Z^{\prime}} \rightarrow \mathrm{TeV}$ since in this case $H^{\prime}$ is too heavy to have an appreciable doublet component. In the opposite limit i.e. when $M_{Z^{\prime}}$ lies close to its lower limit,
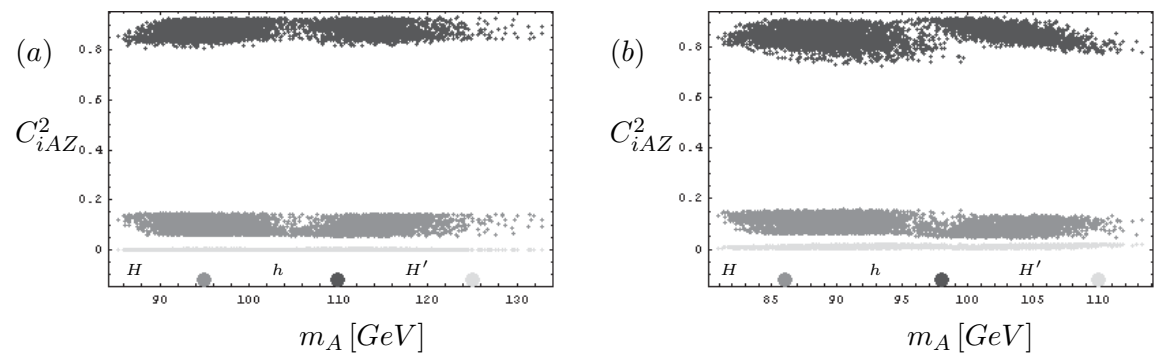

FIG. 3. Variation of $C_{\left(h, H, H^{\prime}\right) A Z}^{2}$ with $m_{A}$ for model I [panel (a)] and model II [panel (b)] after imposing the LEP bounds (15). A comparison with Fig. 2 reveals that $C_{h A Z}^{2} \simeq C_{H Z Z}^{2}, C_{H A Z}^{2} \simeq C_{h Z Z}^{2}$, and $C_{H^{\prime} A Z}^{2} \simeq C_{H^{\prime} Z Z}^{2}$ as expected from discussions in Sec. IV. 
$C_{H^{\prime} Z Z}^{2}$ can compete with $C_{h Z Z}^{2}$ so that correlations among the couplings become too imprecise to compare directly with the MSSM predictions [10,11].

A comparative look at Figs. 2 and 3 reveals the impact of $\mathrm{U}(1)^{\prime}$ charges on Higgs- $Z$ couplings. Indeed, as $\mathrm{U}(1)^{\prime}$ charges are switched from model I to those of model II the shapes and ranges of the allowed domains of couplings change. Obviously, in both models there exist parameter regions where $C_{H^{\prime} Z Z}^{2}$ becomes comparable to $C_{h Z Z}^{2}$. These effects come as no surprise since, as suggested by $Z-Z^{\prime}$ mixing, $M_{Z^{\prime}}$ and the Higgs mass-squared matrix, charge assignments influence various observables. A related point concerns the range of $v_{s}$. Indeed, for keeping $M_{Z^{\prime}}$ within $[0.5,1] \mathrm{TeV}$ interval in both models it is necessary to adjust the range of $v_{s}$ in accord with the $\mathrm{U}(1)^{\prime}$ charges of Higgs fields in the model employed.

Figure 4 illustrates the impact of LEP bounds on the allowed parameter regions. Depicted are variations of Higgs boson masses with $A_{s} / v$ in model I [panels (a) and (c)] and model II [panels (b) and (d)]. The Higgs boson masses in panels (a) and (b) are obtained only when the mass constraints $m_{h} \simeq 98 \mathrm{GeV}$ and $m_{H} \simeq 114 \mathrm{GeV}$ are imposed. In these panels the pseudoscalar mass $m_{A}$ is seen to take values in a rather wide range. What are shown in panels (c) and (d) are the allowed ranges of Higgs boson masses when the constraint that the signal at $98 \mathrm{GeV}$ forms only $\simeq 10 \%$ of the total $[8,10]$ is also included. This constraint, $C_{h Z Z}^{2} \simeq 0.1$, is seen to have a significant effect on the allowed ranges of $m_{A}$. Indeed, the allowed region for
$m_{A}$ is seen to accumulate in mainly two distinct domains: $m_{A}^{\text {high }} \sim m_{H}$ and $m_{A}^{\text {low }} \sim m_{h}$. This classification, however, is not precise at all. First of all, $m_{A}^{\text {high }}$ and $m_{A}^{\text {low }}$ regions are not completely split; there are certain parameter values for which this separation hardly makes sense. Next, in model I, there are regions in the parameter space where $m_{A}^{\text {high }}\left(m_{A}^{\text {low }}\right)$ lies visibly above $m_{H}\left(m_{h}\right)$. Finally, in model II, $m_{A}^{\text {high }}$ $\left(m_{A}^{\text {low }}\right)$ lies significantly below $m_{H}\left(m_{h}\right)$ in almost entire parameter space. This figure is important for revealing the impact of various constraints on the Higgs sector. In general, dynamical natures of $\mu$ and $B$ parameters of the Higgs sector, their correlations with $Z^{\prime}$ mass, and their dependencies on various model parameters (including the one-loop effects computed in [16]) result in certain differences from the MSSM predictions [9-11]. The reasons for these will be clear as we explore correlations among the model parameters in LEP-allowed domains.

Continuing with Fig. 4, one notes that the present LEP data [8] allow for $m_{A}$ to vary over a range that covers both $m_{h}$ and $m_{H}$ such that, given the structure of the allowed domains, there is a rough preference to either $m_{A}^{\text {low }} \sim m_{h}$ or $m_{A}^{\text {high }} \sim m_{H}$. When $m_{A} \sim m_{A}^{\text {low }}$ the pair-production process $e^{+} e^{-} \rightarrow h A$ is kinematically allowed at LEP II energies. Moreover, since $C_{h A Z}^{2} \simeq C_{H Z Z}^{2} \simeq 0.9$ the cross section does not experience any significant suppression with respect to the SM signal except for the fact that the overall signal is suppressed with respect to $H Z$ production due to its $p$ wave nature. The separate LEP experiments have searched for
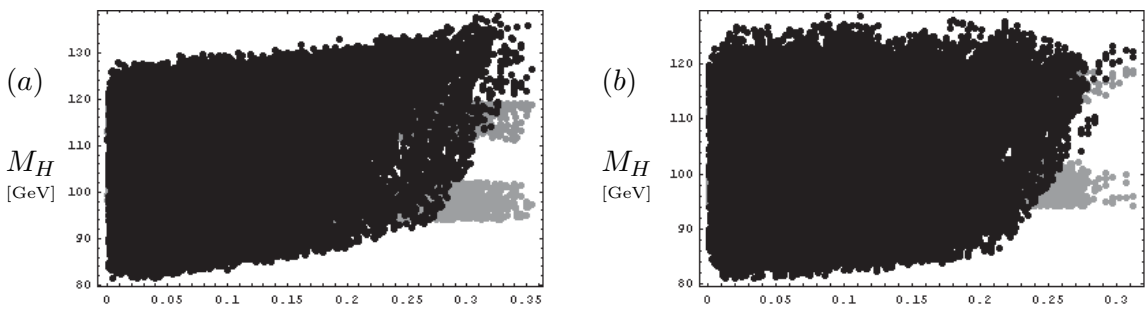

$A_{s} / v$
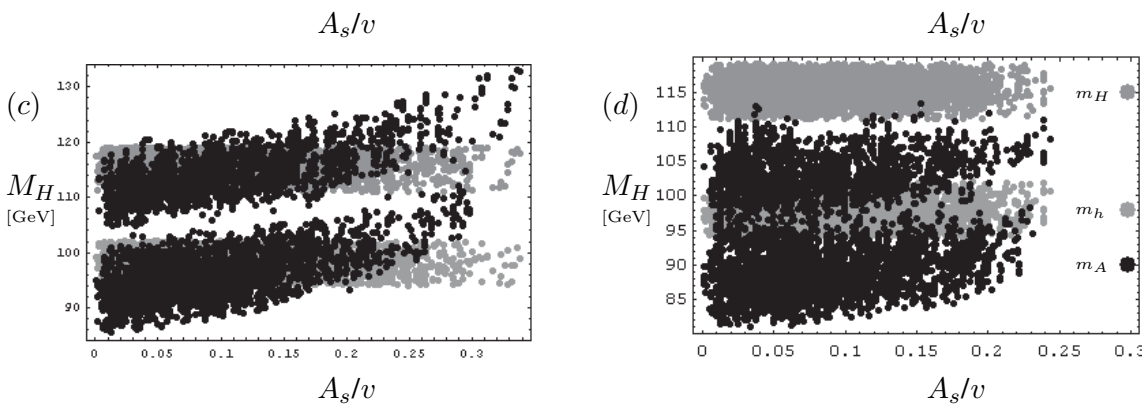

FIG. 4. The impact of LEP bounds on the allowed parameter regions. Depicted are variations of Higgs boson masses [whose shadings are defined by inset in panel (d)] with $A_{s} / v$ in model I [panels (a) and (c)] and model II [panels (b) and (d)]. The Higgs boson masses in panels (a) and (b) are obtained only when the mass constraints $m_{h} \simeq 98 \mathrm{GeV}$ and $m_{H} \simeq 114 \mathrm{GeV}$ are taken into account. In these panels the pseudoscalar mass $m_{A}$ is seen to take values in a rather wide range. The panels (c) and (d) illustrate impact of the constraint that the signal at $98 \mathrm{GeV}$ forms only $\simeq 10 \%$ of the total. This constraint, $C_{h Z z}^{2} \simeq 0.1$, is seen to have a significant effect on the allowed ranges of $m_{A}$. In particular, one notes how $m_{A}$ approximately splits into $m_{A}^{\text {high }}$ (close to $m_{H}$ ) and $m_{A}^{\text {low }}$ (close to $m_{h}$ ) domains. Clearly, these two split regions in which $m_{A}$ could take values vary model to model in shape and separation. 


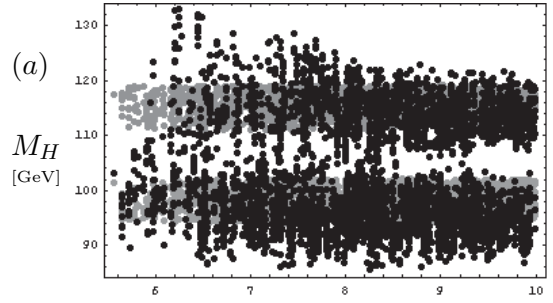

$A_{t} / v$

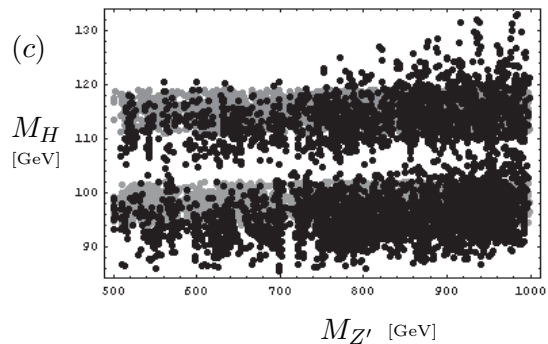

(b)

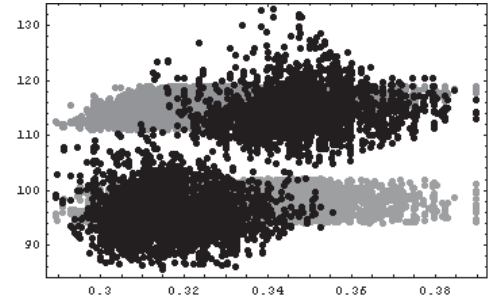

$h_{s}$

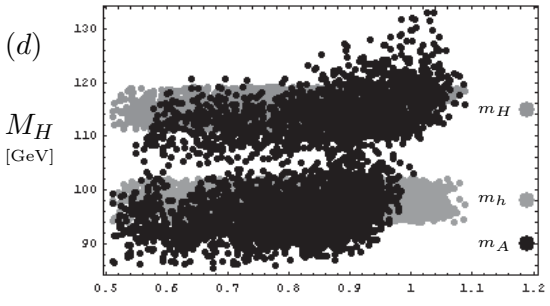

$\mu_{e f f} / v$

FIG. 5. Variations of the Higgs boson masses with various parameters in model I. The $C P$-even Higgs boson $H^{\prime}$ is typically degenerate with $Z^{\prime}$ boson, and its mass is not plotted here. [The inset in panel (d) shows gray levels used for different Higgs boson masses, as in Fig. 4.]

associated $(h, H)+A$ production in the $b \bar{b} b \bar{b}$ and $b \bar{b} \tau^{+} \tau^{-}$ channels. However, a combined analysis of the total LEP sample by all four collaborations is still not available (except in preliminary form [27] which summarizes the status as of summer 2005).

On the other hand, when $m_{A} \sim m_{A}^{\text {high }}$ the pair-production process $e^{+} e^{-} \rightarrow h A$ falls outside the LEP II energy coverage. Moreover, besides $p$-wave suppression, the number of such events should be a small fraction of all such events since $C_{H A Z}^{2} \simeq C_{h Z Z}^{2} \simeq 10 \%$. In either case, the present LEP data favor pseudoscalar Higgs to have a mass roughly equaling $m_{A}^{\text {high }} \sim m_{H}$ or $m_{A}^{\text {low }} \sim m_{h}$.
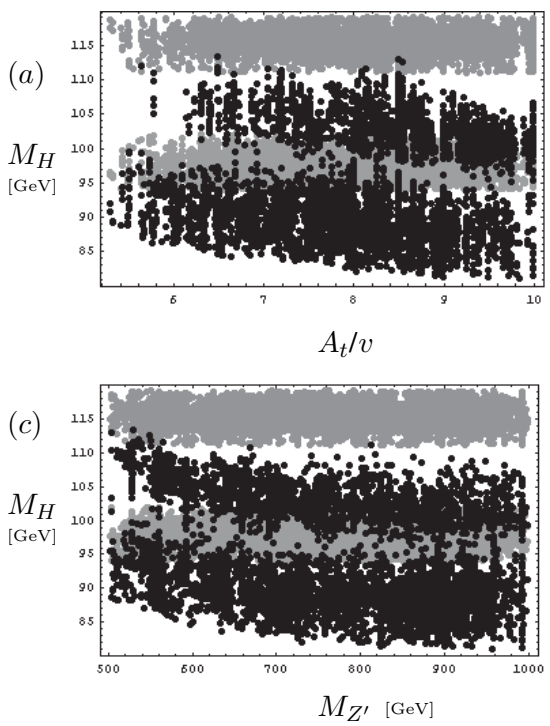

We now continue to explore correlations among various model parameters in light of the LEP bounds (15) on the Higgs boson masses and couplings. Figures 5 and 6 show how Higgs boson masses depend on various parameters in model I and model II, respectively. These figures are particularly useful for determining the allowed ranges of $h_{s}$ and $\mu_{\text {eff }}$ [see the panels (b) and (d) in each figure] while $M_{Z^{\prime}}$ varies in between the two limits and $A_{t}$ respects its upper bound [see panels (a) and (c) in each figure]. Depicted in Fig. 7 are correlations among certain parameters in model I. Furthermore, Table II tabulates precise lower and upper (the numbers in front and inside the

(b)

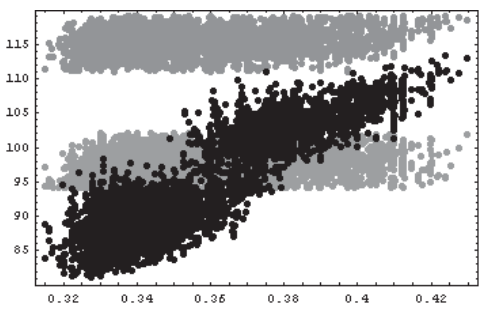

$h_{s}$

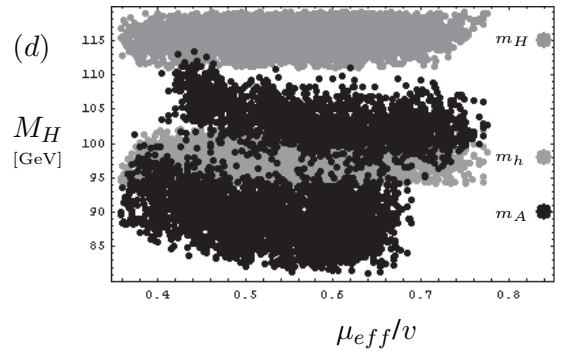

FIG. 6. The same as Fig. 5 but for model II. 
(a)

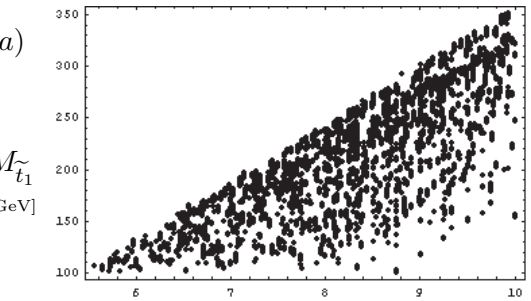

$A_{t} / v$

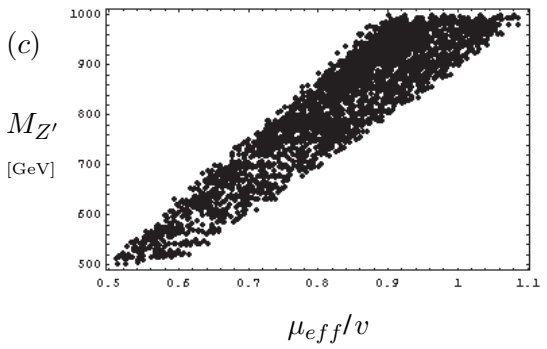

(b)

$A_{t} / v$

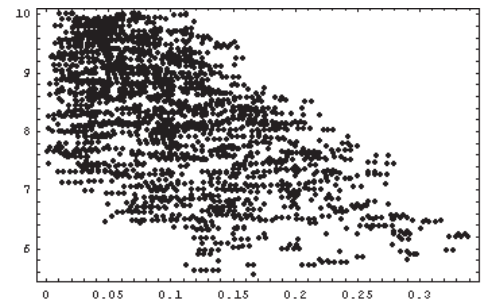

$A_{s} / v$

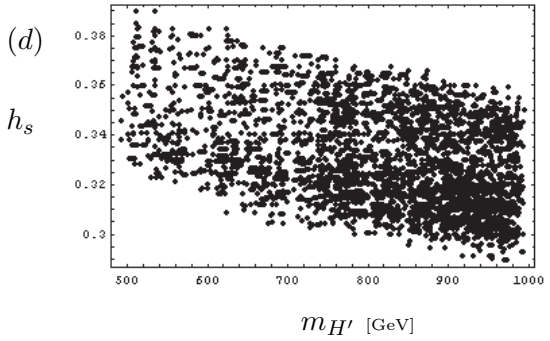

FIG. 7. Correlations among various model parameters. Bounds are similar for model II.

parentheses, respectively) bounds on model parameters and resulting physical particle masses. These numbers are read off from the associated data files. Below we provide a comparative analysis of various parameters illustrated in Figs. 4-7 as well as the limits given in Table II:

(i) Low values of $A_{t}$ are disfavored. Indeed, $A_{t} \geqslant 5 v$ for bounds to be respected. Its minimal value is determined by the lower bound imposed on the light stop mass, $m_{\tilde{t}_{1}} \gtrsim 100 \mathrm{GeV}$ [see Fig. 7(a)]. The precise ranges of $A_{t}$ for each model can be found in Table II. In general, the larger the $A_{t}$ the smaller the $m_{A}$ because radiative correction to $m_{A}$ is proportional to $A_{t}$ and it is negative at large $A_{t}$ where lighter stop weighs well below $M_{Z^{\prime}}$ [16]. Moreover, the light stop mass varies with $A_{t}$ as in panel (a) of Fig. 7. The reason for this behavior is that the soft masses $M_{\tilde{Q}}$ and $M_{\tilde{U}}$ change in the background, and $A_{t}$ is allowed to take larger values as their mean increases. As given in Table II, the light stop mass remains below $\sim 360 \mathrm{GeV}$ and heavy stop weighs above $\sim 660 \mathrm{GeV}$. These masses are well within the range which will be covered by searches at the LHC. (ii) As suggested by Fig. 4 and panel (b) of Fig. 7, high values of $A_{s}$ are disfavored. Indeed, $A_{s}$ is below $v / 3$ in both models (where precise values can be found in Table II). The reason for this is that at large $v_{s}$ (as needed to make $Z^{\prime}$ heavy enough) $A_{s}$ is forced to take small values for making the effective Higgs bilinear mixing $B_{\text {eff }} \propto h_{s} v_{s} A_{s}$ small enough so that the two $C P$-even Higgs bosons (and necessarily the pseudoscalar Higgs) weigh close to $M_{Z}$. In general, the smaller the $A_{s}$ the lighter the $A$ boson [see panels (c) and (d) of Fig. 4] since $m_{A}^{2} \propto$ $B_{\text {eff }}$ at tree level. On the other hand, radiative corrections are enhanced at large $\mu_{\mathrm{eff}} A_{t}$, and thus, $A_{s}$ takes small values at large $A_{t}$ to balance contributions of the one-loop corrections, as suggested by the panel (b) of Fig. 7 (see [16] for dependencies of $m_{A}$ on various parameters).

(iii) As suggested by panels (c) of Figs. 5 and 6, the closer the $M_{Z^{\prime}}$ is to its lower bound the larger the variation in pseudoscalar mass. This is expected since for light $Z^{\prime}$ the singlet vacuum expectation value is lowered and singlet compositions of $h, H$, and $A$ get pronounced. On the other hand, as $M_{Z^{\prime}}$

TABLE II. Allowed ranges of input parameters and predictions for the particle masses in model I and model II.

\begin{tabular}{lccccc}
\hline \hline Inputs & Model I & Model II & Predictions (in GeV) & Model I & Model II \\
\hline$A_{t} / v$ & $5.6(10)$ & $5.3(10)$ & $M \sim$ & $101(352)$ & $100(365)$ \\
$A_{s} / v$ & $0(0.34)$ & $0(0.24)$ & $M_{\sim}^{t_{1}}$ & $665(1130)$ & $658(1202)$ \\
$h_{s}$ & $0.29(0.39)$ & $0.32(0.43)$ & $M_{Z^{\prime}}$ & $501(1000)$ & $502(1000)$ \\
$v_{s} / v$ & $2.1(4.4)$ & $1.4(2.9)$ & $m_{h}$ & $95(101)$ & $95(101)$ \\
$\mu_{\text {eff }} / v$ & $0.51(1.09)$ & $0.36(0.77)$ & $m_{H}$ & $111(119)$ & $111(119)$ \\
$M_{\tilde{Q}} / v$ & $0.6(4)$ & $1.2(4.4)$ & $m_{H^{\prime}}$ & $493(995)$ & $496(996)$ \\
$M_{\tilde{U}} / v$ & $0.6(4)$ & $0.6(4.4)$ & $m_{A}$ & $86(133)$ & $81(113)$ \\
\hline \hline
\end{tabular}


takes on larger values, $m_{A}^{\text {high }}$ and $m_{A}^{\text {low }}$ domains allowed for $m_{A}$ tend to get closer to each other. Therefore, the presence of two roughly distinct regions for $m_{A}$ is related to the extended nature of the Higgs sector (or dynamical nature of the $\mu$ parameter). As it has already been reported in [911], the LEP bounds (15) do not lead to such roughly split regions for $m_{A}$ in the MSSM. Note that gradual decrease of the gap between $m_{A}^{\text {high }}$ and $m_{A}^{\text {low }}$ as $M_{Z^{\prime}}$ increases is a signal of the approach to the MSSM limit. However, one keeps in mind that as $M_{Z^{\prime}}$ increases so does $\mu_{\text {eff }}$ unless $h_{s} A_{s}$ is forced to take small values to keep doublet-dominated Higgs bosons light. This observation is confirmed by panel (d) of Fig. 7. Though hard to confirm experimentally (since experiment will eventually return a specific value for each Higgs boson mass), the aforementioned behavior of $m_{A}$ can be useful for deciding on whether the model under concern is the MSSM or not. This can be accomplished if a certain set of parameters $\mu_{\text {eff }}$, stop masses, soft parameters, etc. is measured and their correlations are confronted with predictions of the model.

(iv) The panels (b) and (d) of Figs. 5 and 6 as well as Table II reveal that $h_{s}$ and $\mu_{\text {eff }} / v$ are restricted to lie within narrow ranges below unity. That these parameters must be bounded is clear from the upper bound on $m_{h}$ given in (8); for given values of $\mathrm{U}(1)^{\prime}$ charges and $g_{Y}^{\prime}$, the most $h_{s}$ can do is to vary within a certain interval in accord with the uncertainty in $m_{h}$ value as well as radiative corrections. Indeed, $h_{s} \in[0.29,0.32]$ in model I and $h_{s} \in[0.39,0.43]$ in model II. Similarly, $\mu_{\text {eff }} \in[0.36,0.51] v$ in model I and $\mu_{\text {eff }} \in[0.77,1.09] v$ in model II. These restrictions arise from lightness of all doublet-dominated Higgs bosons $h, H$, and $A$, and this is realized by rather small values of $h_{s}$. Indeed, heavy $Z^{\prime}$ requires large values of $v_{s}$ with an indirect dependence on $h_{s}$ [see panels (c) and (d) of Fig. 7] whereas the Higgs sector prefers small values of $h_{s} v_{s}$. These observations are further supported by panels (c) and (d) of Fig. 7.

(v) Table II depicts allowed ranges of the model parameters and corresponding predictions for Higgs boson and stop masses when $M_{Z^{\prime}}$ varies in the ranges indicated. Scatter plots of some parameters in this table are provided in Figs. 4-7. For each parameter, the number in parenthesis shows the maximum value and the one in front the minimum value. As it should be clear from the previous figures, some boundaries are already fixed with our choices (e.g. larger values of $A_{t}$ are possible but we keep it below $10 v$ ). In reading this table, it should be kept in mind that we have restricted $M_{Z^{\prime}}$ into a rather conservative range. Indeed, once its upper bound is relaxed $\tan \beta$ will be allowed to swing in a larger range (since then $\left|\alpha_{Z-Z^{\prime}}\right|$ allows for larger values of $\Delta^{2}$ as illustrated in Fig. 1), and it will lead to broadening of the allowed ranges of parameters. However, even in this heavy $Z^{\prime}$ domain, the overall lightness of the Higgs sector will continue to bound $h_{s}$ and $A_{s}$ in ways similar to illustrations given in the figures.

The analysis of the $\mathrm{U}(1)^{\prime}$ parameter space presented in this section takes into account only the LEP bounds (15), and those resulting from the $Z-Z^{\prime}$ mixing. There exist, however, additional indirect bounds from various observables like relic density of neutralinos [28], muon $g-2$ [29], and rare processes [30]. Normally, these additional constraints also must be taken into account for a finer determination of the allowed parameter ranges (as has recently been performed by Hooper and Plehn [11] for the MSSM). In this work we have ignored bounds from such observables though this needs to be confirmed by an explicit calculation.

\section{CONCLUSION}

In this work we have analyzed implications of LEP two light Higgs data on $\mathrm{U}(1)^{\prime}$ models with $M_{Z^{\prime}} \in[0.5,1] \mathrm{TeV}$. We have depicted bounds on various parameters both by scanning of the parameter space and by determining the maximal ranges of the individual parameters. Our results suggest that the model is capable of reproducing the LEP results in wide regions of the parameter space.

We have found that, for $C P$-even Higgs bosons $h$ and $H$ to agree with the LEP data in masses and couplings, (i) the Higgs Yukawa coupling $h_{s}$ and the corresponding soft mass $A_{s}$ are forced to remain bounded in order to keep the Higgs bosons under concern sufficiently light and (ii) the pseudoscalar Higgs boson weighs either close to $m_{h}$ or $m_{H}$ with a finite gap in between. The bounded nature of these parameters stem from our enhanced knowledge about the Higgs boson masses (according to LEP indications). The gap in between $m_{A}^{\text {high }}$ and $m_{A}^{\text {low }}$ bands tends to shrink with increasing $M_{Z^{\prime}}$.

The material presented in this work, in a more general setting, might be regarded as illustrating response of the supersymmetric $\mathrm{U}(1)^{\prime}$ models to constraints enforcing their Higgs sectors to be light. These models, compared to MSSM, are known $[6,16,19]$ to be capable of accommodating larger values for the lightest Higgs boson mass already at tree level. Therefore, their potential to generate smaller values of the lightest Higgs boson masses (as in, for instance, the LEP data [8]) requires certain model parameters to be restricted more strongly than in MSSM or NMSSM. In this sense, results reported in this work might serve as a case study illustrating response of the $\mu$ problem solving models against constraints forcing their Higgs sectors to weigh light. 


\section{ACKNOWLEDGMENTS}

The work of D. D. was partially supported by Turkish Academy of Sciences through GEBIP grant, and by the Scientific and Technical Research Council of Turkey through project 104T503. The work of L.S. was partially supported by the Scientific and Technical Research Council of Turkey.
[1] J. E. Kim and H. P. Nilles, Phys. Lett. 138B, 150 (1984); D. Suematsu and Y. Yamagishi, Int. J. Mod. Phys. A 10, 4521 (1995); M. Cvetic and P. Langacker, Phys. Rev. D 54, 3570 (1996); V. Jain and R. Shrock, hep-ph/9507238; Y. Nir, Phys. Lett. B 354, 107 (1995).

[2] M. Cvetic and S. Godfrey, hep-ph/9504216; P. Langacker, hep-ph/0402203.

[3] J. R. Ellis, J. F. Gunion, H. E. Haber, L. Roszkowski, and F. Zwirner, Phys. Rev. D 39, 844 (1989); S. F. King and P. L. White, Phys. Rev. D 52, 4183 (1995); D. A. Demir, hepph/9902468; Y. Daikoku and D. Suematsu, Phys. Rev. D 62, 095006 (2000).

[4] F. Abe et al. (CDF Collaboration), Phys. Rev. Lett. 79, 2192 (1997); V. M. Abazov et al. (D0 Collaboration), Phys. Rev. Lett. 87, 061802 (2001); A. Abulencia et al. (CDF Collaboration), hep-ex/0507104.

[5] See the note "The $Z^{\prime}$ Searches" by K. S. Babu and C. Kolda in S. Eidelman et al. (Particle Data Group), Phys. Lett. B 592, 1 (2004).

[6] M. Cvetic, D. A. Demir, J. R. Espinosa, L. L. Everett, and P. Langacker, Phys. Rev. D 56, 2861 (1997); 58, 119905(E) (1998).

[7] P. Langacker and J. Wang, Phys. Rev. D 58, 115010 (1998).

[8] G. Abbiendi et al. (ALEPH, DELPHI, L3, and OPAL Collaborations), Phys. Lett. B 565, 61 (2003).

[9] G. L. Kane, T. T. Wang, B.D. Nelson, and L. T. Wang, Phys. Rev. D 71, 035006 (2005).

[10] M. Drees, Phys. Rev. D 71, 115006 (2005).

[11] D. Hooper and T. Plehn, Phys. Rev. D 72, 115005 (2005).

[12] J. L. Hewett and T. G. Rizzo, Phys. Rep. 183, 193 (1989).

[13] M. Cvetic and P. Langacker, Phys. Rev. D 54, 3570 (1996).

[14] C. T. Hill and E. H. Simmons, Phys. Rep. 381, 235 (2003); 390, 553(E) (2004)

[15] J. Erler, P. Langacker, and T. Li, Phys. Rev. D 66, 015002 (2002).

[16] D. A. Demir and L. L. Everett, Phys. Rev. D 69, 015008 (2004).

[17] T. Han, P. Langacker, and B. McElrath, hep-ph/0402064; Phys. Rev. D 70, 115006 (2004).
[18] D. A. Demir and N. K. Pak, Phys. Rev. D 57, 6609 (1998); H. Amini, New J. Phys. 5, 49 (2003).

[19] D. A. Demir, G. L. Kane, and T. T. Wang, Phys. Rev. D 72, 015012 (2005).

[20] F. del Aguila, M. Cvetic, and P. Langacker, Phys. Rev. D 48, R969 (1993); Phys. Rev. D 52, 37 (1995); F. Del Aguila and M. Cvetic, Phys. Rev. D 50, 3158 (1994); A. Leike, Phys. Lett. B 402, 374 (1997); T. Appelquist, B. A. Dobrescu, and A. R. Hopper, Phys. Rev. D 68, 035012 (2003); M. Carena, A. Daleo, B. A. Dobrescu, and T. M. P. Tait, Phys. Rev. D 70, 093009 (2004); J. Kang and P. Langacker, Phys. Rev. D 71, 035014 (2005).

[21] M. Cvetic and P. Langacker, Phys. Rev. D 54, 3570 (1996); Mod. Phys. Lett. A 11, 1247 (1996); D. Suematsu, Phys. Rev. D 59, 055017 (1999).

[22] J. Erler and P. Langacker, Phys. Lett. B 456, 68 (1999); P. Langacker, M. x. Luo, and A. K. Mann, Rev. Mod. Phys. 64, 87 (1992); U. Amaldi et al., Phys. Rev. D 36, 1385 (1987).

[23] K. S. Babu, C. F. Kolda, and J. March-Russell, Phys. Rev. D 57, 6788 (1998).

[24] A. Sopczak, Phys. At. Nucl. 65, 2116 (2002) [Yad. Fiz. 65, 2179 (2002)].

[25] D. A. Demir and N. K. Pak, Phys. Lett. B 411, 292 (1997); Phys. Lett. B 439, 309 (1998).

[26] M. Drees, N. K. Falck, and M. Gluck, Phys. Lett. 167B, 187 (1986).

[27] Peter Igo Kemenes et al. (ALEPH, DELPHI, L3, and OPAL Collaborations), LHWG Report No. Note 200501 (2005). (See http://lp2005.tsl.uu.se/ lp2005/search/pdf/ PAPER-249.pdf.)

[28] V. Barger, C. Kao, P. Langacker, and H. S. Lee, Phys. Lett. B 600, 104 (2004); V. Barger, P. Langacker, and H. S. Lee, Phys. Lett. B 630, 85 (2005).

[29] V. Barger, C. Kao, P. Langacker, and H. S. Lee, Phys. Lett. B 614, 67 (2005).

[30] V. Barger, C. W. Chiang, P. Langacker, and H. S. Lee, Phys. Lett. B 580, 186 (2004); V. Barger, C. W. Chiang, J. Jiang, and P. Langacker, Phys. Lett. B 596, 229 (2004). 